\title{
Editorial
}

\section{Leitthema: Analogien}

$\mathrm{Zu}$ den herausragenden intelligenten Leistungen, die Menschen gegenüber fast allen anderen Lebewesen auszeichnen, gehört der Gebrauch von Analogien. Mit Hilfe von Analogien können neue Probleme, die in der vorliegenden Form bisher noch nicht gelöst wurden, ohne einen aufwendigen, schrittweise ablaufenden Problemlöse- und Lernprozeß bewältigt werden. Manche Probleme sind sogar überhaupt erst mit Hilfe von Analogien gelöst worden, was durch zahlreiche Anekdoten über wissenschaftliche Entdeckungen, die immer wieder zitiert werden, belegt wird. Jedenfalls weisen Analogien ein außerordentliches Potential beim Lösen neuer Probleme auf. Dies ist schon seit längerer Zeit im Bereich psychologischer Forschung thematisiert worden, während in der Informatik eigentlich erst im Laufe der letzten Jahre durch die Forschung zum „Fallbasierten Schließen" Prinzipien analogen und ähnlichkeitsbasierten Problemlösens beim maschinellen Lernen und Problemlösen an Bedeutung gewannen.

Analogien und fallbasiertes Schließen stellen zwei Seiten einer Medaille dar. Während sich Analogien eher mit dem Auffinden und dem Abruf analoger Situationen und der Abbildung zwischen Analoga, aber auch dem darauf basierenden Transfer struktureller Eigenschaften von Analoga zwischen verschiedenen Domänen beschäftigen, geht es in Systemen des fallbasierten Schließens um die Anwendung ähnlicher Situationen und Problemlösungen auf ein aktuelles Problem innerhalb einer Domäne. Die kognitionswissenschaftliche Untersuchung von Analogien beruht in ihrer psychologischen Tradition auf der Untersuchung von Inter-Domänen-Analogien, wobei in der von den amerikanischen Forschungsgruppen um Dedre Gentner und Keith Holyoak geprägten Forschung der letzten 20 Jahre die Untersuchung von Merkmalen eine Rolle spielt, die für unterschiedliche Komponentenprozesse von Analogien relevant sind. Dazu gehören einerseits oberflächliche und semantische Ähnlichkeiten und andererseits strukturelle Übereinstimmungen zwischen Analoga. Aber auch pragmatische Bevorzugungen können, speziell für die Nutzung von Analogien, von Bedeutung sein.
Demgegenüber beschäftigt sich das fallbasierte Schließen vornehmlich mit der ähnlichkeitsbasierten Nutzung früherer Fälle zur Lösung neuer Probleme oder zur Kategorisierung von Fällen innerhalb einer Domäne. Fallbasiertes Schließen geht in seinen ersten Anfängen auf Überlegungen Roger Schanks zum „Dynamischen Gedächtnis" zurück. In den darauf basierenden Arbeiten versuchten seine Schülerinnen und Schüler dann, Schlußfolgerungsmethoden, die für menschliches Problemlösen und Lernen typisch sind, auf maschinelles Lernen und Problemlösen zu übertragen. Inzwischen hat sich der Forschungsbereich „Fallbasiertes Schließen" (insbesondere in Europa) weitgehend von diesen ,psychologisch“ motivierten Ursprüngen gelöst.

Analogien sind damit ein zentrales kognitionswissenschaftliches Thema, wobei nicht vergessen werden soll, daß die in der Linguistik diskutierten Metaphern (als eine in der Sprache manifestierte Form von Analogien) und auch die philosophische Betrachtung von Analogien eine lange Tradition haben. Im deutschsprachigen Raum sind Analogien in den letzten Jahren nur vereinzelt Gegenstand wissenschaftlicher Publikationen gewesen. Auf der letzten Tagung der Gesellschaft für Kognitionswissenschaften im März 1996 gab es einige Beiträge, die sich mit Analogien beschäftigten, allerdings verstreut über inhaltlich sehr unterschiedliche Themenblöcke. Dieses Themenheft soll nun ein Versuch sein, einige Beiträge zum Thema „Analogien“ zu bündeln und einen Überblick über die aktuelle Forschung im deutschsprachigen Raum zu geben.

Mit Bezugnahme auf die Diskussion zur Interdisziplinarität der Kognitionswissenschaft in Heft 6(1) muß festgestellt werden, daß die Beiträge in diesem Heft noch weitgehend von den Teildisziplinen geprägt sind, denen sie entstammen. Auf der einen Seite ist es ein typisch psychologischer Beitrag von Westermann, Hesse, Kauer und Hiemisch, in dem rein empirisch untersucht wird, auf welche Merkmale und Relationen sich die subjektiven Ähnlichkeiten zwischen Analoga zurückführen lassen, die in unterschiedlichen Phasen eines analogen Problemlöseprozesses zum Tragen kommen. Dabei ste- 
hen Analogien zwischen Domänen im Vordergrund. Die Entwicklung eines Modells wird nicht aufgezeigt. Auf der anderen Seite sind es zwei originär informatische Beiträge von Melis und Schmid. Diese Beiträge beschäftigen sich mit mathematischem Beweisen durch Analogie und mit Programmieren durch analoges Schließen. Dabei stehen Analogien innerhalb einer Domäne und die Beschreibung und Entwicklung von Modellen und Systemen für die automatische Anwendung von Analogien im Vordergrund. Empirische Überprüfungen der Modelle werden (noch) nicht durchgeführt.

Ziel sollte es sein, in Zukunft über die den Teildisziplinen verpflichteten Fachbeiträge hinaus zu originär interdisziplinär kognitionswissenschaftlichen Arbeiten zu kommen. Vielleicht kann dieses Heft Anreize zu Ko- operationen schaffen, die für eine Interdisziplinarität förderlich wären. Darüber hinaus kann dieses Heft vielleicht einen Anreiz auch für andere Wissenschaftlerinnen und Wissenschaftler darstellen, sich mit dem spannenden Thema „Analogien“ zu beschäftigen. Auch wenn Analogien ein wissenschaftlich sehr schwieriges Thema sind, was sowohl die empirische Analyse von Analogien beim menschlichen Problemlösen als auch die Modellierung von Analogien in Simulationsmodellen betrifft, so lohnt sich diese Arbeit doch wegen der anfangs aufgeführten außerordentlichen Bedeutung von Analogien für die Leistungsfähigkeit kognitiver Systeme, seien es nun menschliche Problemlöser oder maschinelle Lern- und Problemlösesysteme.

Gerhard Weber 